$\mathrm{SU}-4252-836$

IMSc/2006/08/19

IISc/CHEP/6/06

\title{
Statistics and UV-IR Mixing with Twisted Poincaré Invariance
}

\author{
A. P. Balachandran ${ }^{a *}$, T. R. Govindarajan ${ }^{b \dagger}$, G. Mangano ${ }^{c \ddagger}$ \\ A. Pinzul ${ }^{a \S}$, B. A. Qureshi ${ }^{a}$ and S. Vaidya ${ }^{d \|}$ \\ ${ }^{a}$ Department of Physics, Syracuse University, Syracuse NY, 13244-1130, USA.

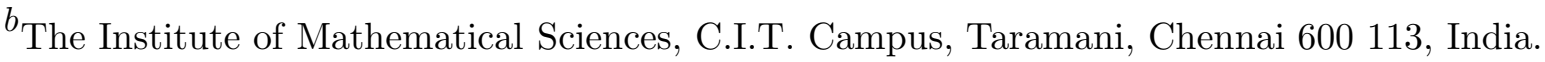 \\ ${ }^{c}$ INFN, Sezione di Napoli and Dipartimento di Scienze Fisiche, \\ Università di Napoli Federico II, Via Cintia, I-80126 Napoli, Italy. \\ ${ }^{d}$ Centre for High Energy Physics, Indian Institute of Science, Bangalore, 560012, India.
}

\begin{abstract}
We elaborate on the role of quantum statistics in twisted Poincaré invariant theories. It is shown that, in order to have twisted Poincaré group as the symmetry of a quantum theory, statistics must be twisted. It is also confirmed that the removal of UV-IR mixing (in the absence of gauge fields) in such theories is a natural consequence.
\end{abstract}

\section{Introduction}

Following the application of Drinfel'd's twist for the Poincaré group on the noncommutative Groenewold-Moyal (GM) plane [1,2], much interest has been generated in the study of its physical consequences. One such consequence pointed out in $[3,4]$ is that the usual statistics

*bal@phy.syr.edu

${ }^{\dagger}$ trg@imsc.res.in

${ }^{\ddagger}$ mangano@na.infn.it

§apinzul@phy.syr.edu

Ibqureshi@phy.syr.edu

" vaidya@cts.iisc.ernet.in 
is not compatible with the twisted action of the Poincaré group. This is in agreement with what is already known in quantum group theory. Among the consequences of this result is the removal of UV-IR mixing [5] in the $S$-matrix in the absence of gauge fields.

Recently there have been claims that this twisting of statistics is unnecessary or even wrong, and that the removal of UV-IR mixing is the result of a wrong choice of interaction. In this note we explain our point of view more clearly, demonstrating that if one wants to retain the twisted Poincaré symmetry in a quantum theory, then one is forced to implement twisted statistics. Secondly, the form of the interaction is dictated by quantum symmetry as well.

The paper is organized as follows. After briefly reviewing the Drinfel'd twist for Poincaré group in the section 2, we elaborate on its implications for quantum statistics in section 3. Section 4 discusses the choice of the correct twisted Lorentz-invariant interaction Hamiltonian. In section 5, we show by an explicit calculation that the correlation functions and hence the $S$-matrix of the noncommutative quantum field theory (NCQFT) with usual statistics are not invariant under the twisted symmetry, while the same are manifestly so for the theory with twisted statistics. Section 6 discusses some issues related to the functional integral for theories with twisted Poincaré symmetry. Section 7 describes the notion of locality in the twisted statistics approach and Section 8 addresses some general issues regarding the tensor products of fields.

\section{The Twist}

The action of a symmetry group on the tensor product of representation spaces carrying the same representation $\rho$ is given by a coproduct $\Delta$ :

$$
g \triangleright(\phi \otimes \chi)=(\rho \otimes \rho) \Delta(g)(\phi \otimes \chi) .
$$

If the representation space happens to be an algebra as well, we further have the compatibility condition

$$
m((\rho \otimes \rho) \Delta(g)(\phi \otimes \chi))=\rho(g) m(\phi \otimes \chi)
$$

where $m$ is the multiplication map.

The GM plane is the algebra $\mathcal{A}_{\theta}$ of functions $f \in \mathbb{R}^{n}$ with the product defined by

$$
f * g=m_{\theta}(f \otimes g)=m_{0}(\mathcal{F} f \otimes g)
$$

where $m_{0}$ is the usual point-wise multiplication,

$$
\mathcal{F}=e^{-\frac{i}{2} \theta^{\mu \nu} P_{\mu} \otimes P_{\nu}}, \quad P_{\mu}=-i \partial_{\mu}
$$

is called the twist element, and this rule for multiplication is often called the star product. Explicitly (2.3) gives

$$
(f * g)(x)=\left.\exp \left(\frac{i}{2} \theta^{\mu \nu} \frac{\partial}{\partial x^{\mu}} \frac{\partial}{\partial y^{\nu}}\right) f(x) g(y)\right|_{x=y} .
$$


The usual coproduct $\Delta_{0}$ on the Poincaré group,

$$
\Delta_{0}(\Lambda)=\Lambda \times \Lambda, \quad \Lambda \in \text { Poincaré group, }
$$

is not compatible with the star product. But a new coproduct $\Delta_{\theta}$ obtained using the twist is compatible, where

$$
\Delta_{\theta}(\Lambda)=\mathcal{F}^{-1} \Delta_{0}(\Lambda) \mathcal{F} .
$$

For details see $[1,2]$. Note that $\Delta_{\theta}(a)=\Delta_{0}(a)$ if $a$ is a translation.

\section{Twisted Statistics}

Twisting the coproduct implies twisting of statistics in quantum theory, as we will argue in this section. This result holds for an $n$-particle quantum mechanical system and also for quantum field theory.

\subsection{Quantum Mechanics}

The wave function of a two-particle system for $\theta^{\mu \nu}=0$ in position representation is a function of two variables, hence lives in $\mathcal{A}_{0} \otimes \mathcal{A}_{0}$, the tensor product of two copies of the algebra of functions on commutative $\mathbb{R}^{n}$, and transforms according to the usual coproduct $\Delta_{0}$. Similarly in noncommutative case, the wavefunction lives in $\mathcal{A}_{\theta} \otimes \mathcal{A}_{\theta}$ and transforms according to the twisted coproduct $\Delta_{\theta}$.

A general element of the tensor product has no particular symmetry. Usually we require that the physical wave functions describing identical particles are either symmetric (bosons) or antisymmetric (fermions). This requires us to work with either the symmetrized or antisymmetrized tensor product

$$
\begin{aligned}
\phi \otimes_{S} \chi & \equiv \frac{1}{2}(\phi \otimes \chi+\chi \otimes \phi), \\
\phi \otimes_{A} \chi & \equiv \frac{1}{2}(\phi \otimes \chi-\chi \otimes \phi)
\end{aligned}
$$

which satisfy

$$
\begin{aligned}
& \phi \otimes_{S} \chi=+\chi \otimes_{S} \phi, \\
& \phi \otimes_{A} \chi=-\chi \otimes_{A} \phi .
\end{aligned}
$$

In a Lorentz-invariant theory, these relations have to hold in all frames of reference. In other words, performing a Lorentz transformation on $\phi \otimes \chi$ and then (anti-)symmetrizing has to be the same as (anti-)symmetrization followed by the Lorentz transformation.

It is not difficult to show that the twisted coproduct (2.7) is not compatible with usual symmetrization/antisymmetrization (3.1, 3.2). To see this, let us write $\mathcal{F}^{-1}$ and $\mathcal{F}$ in the 
Sweedler notation (see for e.g. page 5 of [6]) as

$$
\begin{aligned}
\mathcal{F}^{-1} & =\sum_{\alpha} f^{(1) \alpha} \otimes f_{\alpha}^{(2)}, \quad \mathcal{F}=\sum_{\alpha} \tilde{f}^{(1) \alpha} \otimes \tilde{f}_{\alpha}^{(2)}, \quad \text { with } \\
\mathcal{F}^{-1} \mathcal{F} & =\mathbf{1} \otimes \mathbf{1}=\sum_{\alpha, \beta} f^{(1) \alpha} \tilde{f}^{(1) \beta} \otimes f_{\alpha}^{(2)} \tilde{f}_{\beta}^{(2)} .
\end{aligned}
$$

Under a Lorentz transformation $\Lambda$,

$$
\begin{aligned}
\Lambda: \phi \otimes \chi & \longrightarrow(\rho \otimes \rho) \Delta_{\theta}(\Lambda)(\phi \otimes \chi) \\
& =\sum_{\alpha, \beta} \rho\left(f^{(1) \alpha} \Lambda \tilde{f}^{(1) \beta}\right) \phi \otimes \rho\left(f_{\alpha}^{(2)} \Lambda \tilde{f}_{\beta}^{(2)}\right) \chi
\end{aligned}
$$

Subsequent symmetrization/antisymmetrization gives us

$$
\sum_{\alpha, \beta}\left(\rho\left(f^{(1) \alpha} \Lambda \tilde{f}^{(1) \beta}\right) \phi \otimes \rho\left(f_{\alpha}^{(2)} \Lambda \tilde{f}_{\beta}^{(2)}\right) \chi \pm \rho\left(f_{\alpha}^{(2)} \Lambda \tilde{f}_{\beta}^{(2)}\right) \chi \otimes \rho\left(f^{(1) \alpha} \Lambda \tilde{f}^{(1) \beta}\right) \phi\right)
$$

whereas

$$
\begin{aligned}
& (\rho \otimes \rho) \Delta_{\theta}(\Lambda)\left(\phi \otimes_{S, A} \chi\right)= \\
& \quad \sum_{\alpha^{\prime} \beta}\left(\rho\left(f^{(1) \alpha} \Lambda \tilde{f}^{(1) \beta}\right) \phi \otimes \rho\left(f_{\alpha}^{(2)} \Lambda \tilde{f}_{\beta}^{(2)}\right) \chi \pm \rho\left(f^{(1) \alpha} \Lambda \tilde{f}^{(1) \beta}\right) \chi \otimes \rho\left(f_{\alpha}^{(2)} \Lambda \tilde{f}_{\beta}^{(2)}\right) \phi\right)
\end{aligned}
$$

which is not the same as (3.8) [See [4] for the same proof which avoids Sweedler notation.]. The origin of this difference can be traced to the fact that the coproduct is not cocommutative except when $\theta^{\mu \nu}=0$.

There is another way to phrase this compatibility (or lack thereof) of Lorentz transformations and symmetrization. Let $\tau_{0}$ be the statistics (flip) operator associated with exchange:

$$
\tau_{0}(\phi \otimes \chi)=\chi \otimes \phi
$$

In usual quantum theory, we have the axiom that $\tau_{0}$ is superselected, i.e., all the observables commute with $\tau_{0}$. What this means is that no operator in the physical Hilbert space can change statistics. In particular the quantum operators that implement Lorentz symmetry must commute with the statistics operator. Also all the states in a given superselection sector are eigenstates of $\tau_{0}$ with the same eigenvalue. Given an element $\phi \otimes \chi$ of the tensor product, the physical Hilbert spaces can be constructed from the elements

$$
\left(\frac{1 \pm \tau_{0}}{2}\right)(\phi \otimes \chi)
$$

As is obvious from eq (3.83.9),

$$
\tau_{0} \Delta_{\theta}(\Lambda) \neq \Delta_{\theta}(\Lambda) \tau_{0}
$$


showing that the usual statistics is not compatible with the coproduct. But notice that the new statistics operator

$$
\tau_{\theta} \equiv \mathcal{F}^{-1} \tau_{0} \mathcal{F}, \quad \tau_{\theta}^{2}=\mathbf{1} \otimes \mathbf{1}
$$

does commute with the twisted coproduct. The states constructed according to

$$
\phi \otimes_{S_{\theta}} \chi \equiv\left(\frac{1+\tau_{\theta}}{2}\right)(\phi \otimes \chi), \quad \phi \otimes_{A_{\theta}} \chi \equiv\left(\frac{1-\tau_{\theta}}{2}\right)(\phi \otimes \chi)
$$

form the physical two-particle Hilbert spaces of (generalized) bosons and fermions and obey twisted statistics.

For plane waves $e_{p}(x)=e^{-i p \cdot x}$ we get

$$
\begin{aligned}
\left(\frac{1 \pm \tau_{\theta}}{2}\right)\left(e_{p} \otimes e_{q}\right) & \equiv e_{p} \otimes_{S_{\theta}, A_{\theta}} e_{q}= \pm e^{-i p_{\mu} \theta^{\mu \nu} q_{\nu}} e_{q} \otimes_{S_{\theta}, A_{\theta}} e_{p}, \\
\left(e_{p} \otimes_{S_{\theta}, A_{\theta}} e_{q}\right)\left(x_{1}, x_{2}\right) & = \pm e^{-i \frac{\partial}{\partial x_{1}^{\mu}} \theta^{\mu \nu} \frac{\partial}{\partial x_{2}^{\nu}}}\left(e_{p} \otimes_{S_{\theta}, A_{\theta}} e_{q}\right)\left(x_{2}, x_{1}\right) .
\end{aligned}
$$

Using the anti-symmetry of $\theta^{\mu \nu}, \tau_{\theta}$ may also be equivalently written as

$$
\tau_{\theta}=\mathcal{F}^{-2} \tau_{0} .
$$

This form of $\tau_{\theta}$ allows to make contact with quantum group theory, and identifies $\mathcal{F}^{-2}$ as the corresponding $R$-matrix.

\subsection{Statistics of Quantum Fields}

A quantum field on evaluation at a spacetime point (or more generally on pairing with a test function) gives an operator acting on a Hilbert space. A field at $x_{1}$ acting on the vacuum gives a one-particle state centered at $x_{1}$. When we write $\Phi\left(x_{1}\right) \Phi\left(x_{2}\right)$ we mean $(\Phi \otimes \Phi)\left(x_{1}, x_{2}\right)$. Acting on the vacuum we generate a two-particle state, where one particle is centered at $x_{1}$ and the other at $x_{2}$. (We retain just the creation operator part of $\Phi$ here.) Notice that it just involves evaluation of the two functions in the tensor product and not a multiplication map as we get a function of two variables. On the other hand the star product is a map from $\mathcal{A}_{\theta} \otimes \mathcal{A}_{\theta}$ to $\mathcal{A}_{\theta}$ and gives a function of a single variable. Hence there is no reason a priori to put a star-like operation between $\Phi\left(x_{1}\right) \Phi\left(x_{2}\right)$. We will have more to say about this in Section 8 .

If $a_{p}$ is the annihilation operator of the second-quantized field $\Phi(x)$, we want, as in standard quantum field theory,

$$
\begin{aligned}
\left\langle 0\left|\Phi^{(-)}(x) a_{p}^{\dagger}\right| 0\right\rangle & =e_{p}(x) \\
\frac{1}{2}\left\langle 0\left|\Phi^{(-)}\left(x_{1}\right) \Phi^{(-)}\left(x_{2}\right) a_{q}^{\dagger} a_{p}^{\dagger}\right| 0\right\rangle & =\left(\frac{\mathbf{1} \pm \tau_{\theta}}{2}\right)\left(e_{p} \otimes e_{q}\right)\left(x_{1}, x_{2}\right) \\
& \equiv\left(e_{p} \otimes_{S_{\theta}, A_{\theta}} e_{q}\right)\left(x_{1}, x_{2}\right)
\end{aligned}
$$


[We suppress spin indices. Also here we retain only the annihilation part of the field in $\left.\Phi^{(-)}\right]$. Note the reversal of $p$ and $q$ from LHS to RHS of (3.19). This is the standard prescription used to establish the connection between quantum field operators and (multi)particle wavefunctions. The correctness of this prescription can be verified by applying it to the fermionic case, for $\theta^{\mu \nu}=0$.

This compatibility between twisted statistics and Poincaré invariance has profound consequences for commutation relations. For example when the states are labeled by momenta, we have, from exchanging $p$ and $q$ in (3.19)

$$
|p, q\rangle_{S_{\theta}, A_{\theta}}= \pm e^{i \theta^{\mu \nu} p_{\mu} q_{\nu}}|q, p\rangle_{S_{\theta}, A_{\theta}}
$$

This is the origin of the commutation relation

$$
a_{p}^{\dagger} a_{q}^{\dagger}= \pm e^{i \theta^{\mu \nu} p_{\mu} q_{\nu}} a_{q}^{\dagger} a_{p}^{\dagger} .
$$

The adjoint relation

$$
a_{p} a_{q}= \pm e^{i \theta^{\mu \nu} p_{\mu} q_{\nu}} a_{q} a_{p}
$$

also follows from the complex conjugate of (3.19) on using (3.16).

The statistics induced on the free quantum fields by (3.19) is given, on using (3.16), by

$$
\Phi^{(-)}\left(x_{1}\right) \Phi^{(-)}\left(x_{2}\right)= \pm e^{i \theta^{\mu \nu} \frac{\partial}{\partial x_{2}^{\mu}} \frac{\partial}{\partial x_{1}^{\nu}}} \Phi^{(-)}\left(x_{2}\right) \Phi^{(-)}\left(x_{1}\right) .
$$

Any quantization has to be compatible with the above statistics of the fields.

So far we have had no occasion to use the algebraic properties of $\mathcal{A}_{\theta}$. All we have used is the assumption that the symmetry of the theory is the twisted Poincaré group symmetry. That, of course, was forced on us from automorphism properties of $\mathcal{A}_{\theta}$.

\section{Choice of Interaction Hamiltonian}

It was claimed by [7] that the removal of UV-IR mixing in noncommutative theories may be due to an inappropriate choice of the interaction Hamiltonian. Here we point out that our choice of the Hamiltonian is forced on us from the requirement of twisted Poincaré invariance.

The interaction Hamiltonian is built out of fields. We need a multiplication map to write down a Hamiltonian density starting from fields, as it is a scalar function of just one variable. Also in order to have twisted Poincaré invariance, one has to ensure that the Hamiltonian density transforms like a scalar field. This will only happen if we choose a star product (twisted multiplication map) between the fields to write down the Hamiltonian density. Hence a generic interaction Hamiltonian density involving just one hermitean spin zero field (for simplicity) is

$$
\mathcal{H}_{I}(x)=\Phi(x) * \Phi(x) * \cdots * \Phi(x)
$$

where $\Phi(x)$ obeys twisted statistics. This form of Hamiltonian and the twisted statistics of the fields is all that is needed to show that there is no UV-IR mixing in this theory $[3,5]$. 


\section{On the Invariance of Correlation Functions}

i) The Twisted Action on the Tensor Product of Plane Waves:

As a preliminary to the calculations, we first consider the actions of the twisted coproduct of the Poincaré group on the tensor products of plane waves.

On a single plane wave, the Lorentz transformation $\Lambda$ and translation $P_{\mu}$ acts according to

$$
\begin{aligned}
\left(\Lambda e_{p}\right)(x) & =e_{p}\left(\Lambda^{-1} x\right)=e_{\Lambda p}(x), \\
\left(P_{\mu} e_{p}\right)(x) & =-p_{\mu} e_{p}(x)
\end{aligned}
$$

where we used $\Lambda^{-1}=\Lambda^{T}$ and $P_{\mu}=-i \partial_{\mu}$. Hence

$$
\Lambda e_{p}=e_{\Lambda p}, \quad \partial_{\mu} e_{p}=-i p_{\mu} e_{p} .
$$

Let $U$ denote the representation of the (enveloping algebra of the) Poincaré group on arbitrary tensor products of plane waves. The latter respond to translations in the usual manner, so we focus on Lorentz transformations $\Lambda$. On $e_{k}$, the action of $U(\Lambda)$ is as in (5.1):

$$
U(\Lambda) e_{k}=e_{\Lambda k} .
$$

On $e_{k_{1}} \otimes e_{k_{2}}$, we must find the action using the coproduct:

$$
\begin{aligned}
U(\Lambda) e_{k_{1}} \otimes e_{k_{2}} & =\Delta_{\theta}(\Lambda) e_{k_{1}} \otimes e_{k_{2}} \\
& =e^{-\frac{i}{2} \partial_{\mu} \theta^{\mu \nu} \otimes \partial_{\nu}}(\Lambda \otimes \Lambda) e^{\frac{i}{2} \partial_{\mu} \theta^{\mu \nu} \otimes \partial_{\nu}} e_{k_{1}} \otimes e_{k_{2}} \\
& =e_{\Lambda k_{1}} \otimes \underbrace{e^{-\frac{1}{2}\left(\Lambda k_{1}\right)_{\mu} \theta^{\mu \nu} \partial_{\nu}} \Lambda e^{\frac{1}{2} k_{1 \mu} \theta^{\mu \nu} \partial_{\nu}}}_{\Lambda_{1}} e_{k_{2}} \\
& =e^{\frac{i}{2} k_{1} \cdot \delta_{\Lambda} \theta \cdot k_{2}} e_{\Lambda k_{1}} \otimes e_{\Lambda k_{2}},
\end{aligned}
$$

where

$$
k_{1} \cdot \delta_{\Lambda} \theta \cdot k_{2} \equiv k_{1 \mu}\left(\delta_{\Lambda} \theta\right)^{\mu \nu} k_{2 \nu}, \quad \delta_{\Lambda} \theta \equiv \Lambda^{-1} \theta \Lambda-\theta .
$$

The action on $e_{k_{1}} \otimes e_{k_{2}} \otimes e_{k_{3}}$ is found using the coproduct on $\Lambda_{1}$ :

$$
\begin{aligned}
\Delta_{\theta}\left(\Lambda_{1}\right)= & \left(e^{-\frac{1}{2}\left(\Lambda k_{1}\right)_{\mu} \theta^{\mu \nu} \partial_{\nu}} \otimes e^{-\frac{1}{2}\left(\Lambda k_{1}\right)_{\mu} \theta^{\mu \nu} \partial_{\nu}}\right)\left(e^{-\frac{i}{2} \partial_{\mu} \theta^{\mu \nu} \otimes \partial_{\nu}} \Lambda \otimes \Lambda e^{\frac{i}{2} \partial_{\mu} \theta^{\mu \nu} \otimes \partial_{\nu}}\right) \times \\
& \times\left(e^{\frac{1}{2} k_{1 \mu} \theta^{\mu \nu} \partial_{\nu}} \otimes e^{\frac{1}{2} k_{1 \mu} \theta^{\mu \nu} \partial_{\nu}}\right) .
\end{aligned}
$$

It gives

$$
U(\Lambda) e_{k_{1}} \otimes e_{k_{2}} \otimes e_{k_{3}}=e_{\Lambda k_{1}} \otimes \Delta_{\theta}\left(\Lambda_{1}\right)\left(e_{k_{2}} \otimes e_{k_{3}}\right)
$$

where

$$
\begin{aligned}
\Delta_{\theta}\left(\Lambda_{1}\right)\left(e_{k_{2}} \otimes e_{k_{3}}\right) & =e^{\frac{i}{2} k_{1} \cdot \delta_{\Lambda} \theta \cdot k_{2}} e_{\Lambda k_{2}} \otimes \Lambda_{2} e_{k_{3}}, \\
\Lambda_{2} & =e^{-\frac{1}{2}\left(\Lambda k_{1}+\Lambda k_{2}\right)_{\mu} \theta^{\mu \nu} \partial_{\nu}} \Lambda e^{\frac{1}{2}\left(k_{1}+k_{2}\right)_{\mu} \theta^{\mu \nu} \partial_{\nu}} .
\end{aligned}
$$


Thus

$$
U(\Lambda) e_{k_{1}} \otimes e_{k_{2}} \otimes e_{k_{3}}=e^{\frac{i}{2} k_{1} \cdot \delta_{\Lambda} \theta \cdot k_{2}+\frac{i}{2}\left(k_{1}+k_{2}\right) \cdot \delta_{\Lambda} \theta \cdot k_{3}} e_{\Lambda k_{1}} \otimes e_{\Lambda k_{2}} \otimes e_{\Lambda k_{3}}
$$

The action on $e_{k_{1}} \otimes e_{k_{2}} \otimes e_{k_{3}} \otimes e_{k_{4}}$ is found by splitting $\Lambda_{2}$ again with a $\Delta_{\theta}$. In this way we see that in general,

$U(\Lambda) e_{k_{1}} \otimes e_{k_{2}} \ldots \otimes e_{k_{N}}=e^{\frac{i}{2} k_{1} \cdot \delta_{\Lambda} \theta \cdot k_{2}+\frac{i}{2}\left(k_{1}+k_{2}\right) \cdot \delta_{\Lambda} \theta \cdot k_{3}+\ldots\left(k_{1}+k_{2} \ldots+k_{N-1}\right) \cdot \delta_{\Lambda} \cdot \theta k_{N}} e_{\Lambda k_{1}} \otimes e_{\Lambda k_{2}} \ldots \otimes e_{\Lambda k_{N}}$.

ii) Correlation Functions of NCQFT with Untwisted Statistics:

Consider the scalar field theory on the GM plane with the Lagrangian (density)

$$
\mathcal{L}_{*}=\frac{1}{2} \partial_{\mu} \Phi * \partial^{\mu} \Phi-\frac{1}{2} m^{2} \Phi * \Phi-\frac{\lambda}{4 !} \Phi * \Phi * \Phi * \Phi,
$$

where $\Phi^{\dagger}=\Phi$. Since statistics is not twisted, the annihilation and creation operators $c_{p}, c_{p}^{\dagger}$ of $\Phi$ are those for $\theta^{\mu \nu}=0$.

The correlation functions of (5.11) are not Lorentz-invariant under the twisted coproduct. It is enough to prove this result for the free field theory where $\lambda=0$.

The correlation functions for the product of an odd number of fields is zero. We show now that the four-point function is not Lorentz-invariant under the twisted coproduct. That can be adapted to show that the two-point function is Lorentz invariant. (Translational invariance is preserved by both untwisted and twisted statistics.)

The scalar field has the mode expansion

$$
\Phi(x)=\int \frac{d^{3} p}{(2 \pi)^{3 / 2}\left(2 p_{0}\right)}\left(c_{p} e_{p}(x)+c_{p}^{\dagger} e_{-p}(x)\right)
$$

where $p_{0}=+\sqrt{|\vec{p}|^{2}+m^{2}}$ and $c_{p}$ and $c_{p}^{\dagger}$ are the annihilation-creation operators for $\theta^{\mu \nu}=0$ :

$$
\begin{aligned}
& {\left[c_{p}, c_{k}\right]=0=\left[c_{p}^{\dagger}, c_{k}^{\dagger}\right]} \\
& {\left[c_{k}, c_{k}^{\dagger}\right]=2 p_{0} \delta^{3}(p-k)}
\end{aligned}
$$

The four point function in this case, with no statistics twist, is

$$
\begin{aligned}
\left\langle 0\left|\Phi\left(x_{1}\right) \Phi\left(x_{2}\right) \Phi\left(x_{3}\right) \Phi\left(x_{4}\right)\right| 0\right\rangle= & D\left(x_{1}-x_{2}\right) D\left(x_{3}-x_{4}\right)+D\left(x_{1}-x_{3}\right) D\left(x_{2}-x_{4}\right) \\
& +D\left(x_{1}-x_{4}\right) D\left(x_{2}-x_{3}\right) \\
\equiv & I+I I+I I I \\
D(x)= & \int \frac{d^{3} p}{(2 \pi)^{3}\left(2 p_{0}\right)} e^{-i p \cdot x}=D(\Lambda x) .
\end{aligned}
$$

We now show that $I$ and $I I I$ are invariant (for the twisted coproduct), but not $I I$.

Consider $I$ :

$I=\frac{1}{(2 \pi)^{6}} \int\left(\prod_{i} \frac{d^{3} p_{i}}{\left(2 p_{i 0}\right)}\right) e_{p_{1}}\left(x_{1}\right) e_{-p_{2}}\left(x_{2}\right) e_{p_{3}}\left(x_{3}\right) e_{-p_{4}}\left(x_{4}\right)\left(2 p_{10}\right)\left(2 p_{30}\right) \delta^{3}\left(p_{1}-p_{2}\right) \delta^{3}\left(p_{3}-p_{4}\right)$. 
Applying (5.10) with $k_{1}=p_{1}, k_{2}=-p_{2}, k_{3}=p_{3}, k_{4}=-p_{4}$, we find that the phase in (5.10) becomes 1 because of the $\delta$-functions and that

$$
\Lambda: I \rightarrow D\left(\Lambda^{-1}\left(x_{1}-x_{2}\right)\right) D\left(\Lambda^{-1}\left(x_{3}-x_{4}\right)\right)=I .
$$

A similar calculation shows the Lorentz invariance of $I I I$.

Now consider $I I$ :

$I I=\frac{1}{(2 \pi)^{6}} \int\left(\prod_{i} \frac{d^{3} p_{i}}{\left(2 p_{i 0}\right)}\right) e_{p_{1}}\left(x_{1}\right) e_{p_{2}}\left(x_{2}\right) e_{-p_{3}}\left(x_{3}\right) e_{-p_{4}}\left(x_{4}\right)\left(2 p_{10}\right)\left(2 p_{20}\right) \delta^{3}\left(p_{1}-p_{3}\right) \delta^{3}\left(p_{2}-p_{4}\right)$.

So with $k_{1}=p_{1}, k_{2}=p_{2}, k_{3}=-p_{3}, k_{4}=-p_{4}$ the phase becomes $e^{\frac{i}{2} p_{1} \cdot \delta_{\Lambda} \theta \cdot p_{2}}$ and

$$
\Lambda: I I \rightarrow \int \frac{d^{3} p_{1} d^{3} p_{2}}{(2 \pi)^{6}\left(2 p_{10}\right)\left(2 p_{20}\right)} e^{i p_{1} \cdot \delta_{\Lambda} \theta \cdot p_{2}} e^{i\left(\Lambda p_{1}\right) \cdot\left(x_{1}-x_{3}\right)} e^{i\left(\Lambda p_{2}\right) \cdot\left(x_{2}-x_{4}\right)} \neq I I .
$$

It is not Lorentz-invariant.

iii) Correlation Functions of NCQFT with Twisted Statistics:

In this case the free field is

$$
\Phi(x)=\int \frac{d^{3} p}{(2 \pi)^{3 / 2}\left(2 p_{0}\right)}\left(a_{p} e_{p}(x)+a_{p}^{\dagger} e_{-p}(x)\right) .
$$

Let $\mathcal{P}_{\mu}$ be the Fock space momentum operator:

$$
\mathcal{P}_{\mu}=\int \frac{d^{3} p}{2 p_{0}} p_{\mu} c_{p}^{\dagger} c_{p}
$$

Then, as shown in $[4,8]$, the operators $a_{p}, a_{p}^{\dagger}$ can be written as follows:

$$
a_{p}=c_{p} e^{-\frac{i}{2} p_{\mu} \theta^{\mu \nu} \mathcal{P}_{\nu}}, \quad a_{p}^{\dagger}=c_{p}^{\dagger} e^{+\frac{i}{2} p_{\mu} \theta^{\mu \nu} \mathcal{P}_{\nu}} .
$$

Using (5.16) and (5.18), we calculate the four-point function with twisted statistics:

$$
\begin{aligned}
& \left\langle 0\left|\Phi\left(x_{1}\right) \Phi\left(x_{2}\right) \Phi\left(x_{3}\right) \Phi\left(x_{4}\right)\right| 0\right\rangle=I+I I I+ \\
& \frac{1}{(2 \pi)^{6}} \int \prod_{i} \frac{d^{3} p_{i}}{\left(2 p_{i 0}\right)} e^{i p_{1 \mu} \theta^{\mu \nu} p_{2 \nu}} e_{p_{1}}\left(x_{1}\right) e_{-p_{2}}\left(x_{2}\right) e_{p_{3}}\left(x_{3}\right) e_{-p_{4}}\left(x_{4}\right) \times \\
& \left(2 p_{10}\right)\left(2 p_{20}\right) \delta^{3}\left(p_{1}-p_{3}\right) \delta^{3}\left(p_{2}-p_{4}\right) . \\
\equiv & I+I I I+I I^{\prime}
\end{aligned}
$$

where $I$ and $I I I$ are Poincaré invariant as shown before. As for $I I^{\prime}$, we find, using (5.10) with $k_{1}=p_{1}, k_{2}=-p_{2}, k_{3}=p_{3}, k_{4}=-p_{4}$ and the $\delta$-functions,

$$
\begin{aligned}
& \Lambda: I I^{\prime} \rightarrow \frac{1}{(2 \pi)^{6}} \int \prod_{i} \frac{d^{3} p_{i}}{\left(2 p_{i 0}\right)} e_{\Lambda p_{1}}\left(x_{1}\right) e_{-\Lambda p_{2}}\left(x_{2}\right) e_{\Lambda p_{3}}\left(x_{3}\right) e_{-\Lambda p_{4}}\left(x_{4}\right) \\
& e^{i p_{1 \mu} \theta^{\mu \nu} p_{2 \nu}} e^{i p_{1} \cdot \delta_{\Lambda} \theta \cdot p_{2}}\left(2 p_{10}\right)\left(2 p_{20}\right) \delta^{3}\left(p_{1}-p_{3}\right) \delta^{3}\left(p_{2}-p_{4}\right) .
\end{aligned}
$$


Since

$$
e^{i p_{1 \mu} \theta^{\mu \nu} p_{2 \nu}} e^{i p_{1} \cdot \delta_{\Lambda} \theta \cdot p_{2}}=e^{i\left(\Lambda p_{1}\right)_{\mu} \theta^{\mu \nu}\left(\Lambda p_{2}\right)_{\nu}}
$$

the Poincaré invariance of $I I^{\prime}$ also follows. The phase $e^{i p_{1 \mu} \theta^{\mu \nu} p_{2 \nu}}$ in (5.23) which comes from twisted statistics is essential to reach this conclusion.

\section{Functional Integral}

We saw above that in order to have twisted Poincaré invariance in a quantum theory, we must also have twisted statistics. This has implications for a functional integral formulation of the quantum theory too. This is because statistics of the fields is an input in a functional integral. For example, in the case of usual fermions, statistics is not derived from functional integral, but is rather inferred from other considerations and then built into the functional integral by use of anticommuting classical fields.

Similarly, in order to construct a functional integral which gives a twisted Poincaré invariant quantum theory, we must use the correct statistics as an input and construct the functional integral out of classical fields which obey the twisted statistics. In particular its full measure consists of tensor products of individual measures at different points and the individual measures must obey twisted statistics among themselves in order for the total measure to be Poincaré invariant. This again is in analogy to the case of fermions, where individual measures anticommute among themselves. We will not go here into the full details of the construction of the functional integral which gives the twisted quantum field theory. It has been done by Oeckl [10]. It will suffice here to show that the conventional functional integral does not give a twisted Poincaré invariant theory.

The following functional integral was considered by [9] and claimed to be twist-Poincaré invariant:

$$
W=\int \prod_{x} \mathcal{D}(\phi(x)) e^{i \int d^{4} x \mathcal{L}_{*}(x)}
$$

where $\mathcal{L}_{*}$ is for example the star-Lagrangian (density)

$$
\mathcal{L}_{*}(x)=\frac{1}{2} \partial_{\mu} \phi(x) * \partial^{\mu} \phi(x)-\frac{1}{2} m^{2} \phi(x) * \phi(x)-\frac{\lambda}{4 !} \phi(x) * \phi(x) * \phi(x) * \phi(x)
$$

and $\mathcal{D}(\phi(x))$ is the usual measure.

With the functional integral defined with this measure, we obtain conventional quantization of noncommutative field theory with no statistics twist, and its Feynman rules.

But this measure is not invariant under the twisted Poincaré group. We can show this by a simple argument.

Consider

$$
\begin{aligned}
\int \prod_{x} \mathcal{D}(\phi(x)) \phi\left(x_{1}\right) & \phi\left(x_{2}\right) \phi\left(x_{3}\right) \phi\left(x_{4}\right) e^{i \int d^{4} x \mathcal{L}_{*}(x)} \\
& =\left\langle 0\left|T\left\{\Phi\left(x_{1}\right) \Phi\left(x_{2}\right) \Phi\left(x_{3}\right) \Phi\left(x_{4}\right)\right\}\right| 0\right\rangle .
\end{aligned}
$$


It is enough to consider $\lambda=0$. Let us suppose for convenience that $x_{1}^{0}>x_{2}^{0}>x_{3}^{0}>x_{4}^{0}$. Then

$$
\left\langle 0\left|T\left\{\Phi\left(x_{1}\right) \Phi\left(x_{2}\right) \Phi\left(x_{3}\right) \Phi\left(x_{4}\right)\right\}\right| 0\right\rangle=\left\langle 0\left|\Phi\left(x_{1}\right) \Phi\left(x_{2}\right) \Phi\left(x_{3}\right) \Phi\left(x_{4}\right)\right| 0\right\rangle .
$$

which is the same as (5.14). But we saw above that

$$
\left\langle 0\left|\Phi\left(x_{1}\right) \Phi\left(x_{2}\right) \Phi\left(x_{3}\right) \Phi\left(x_{4}\right)\right| 0\right\rangle \neq \Lambda \triangleright\left\langle 0\left|\Phi\left(x_{1}\right) \Phi\left(x_{2}\right) \Phi\left(x_{3}\right) \Phi\left(x_{4}\right)\right| 0\right\rangle .
$$

Hence it follows that

$$
\begin{aligned}
\int \prod_{x} \mathcal{D}(\phi(x)) \phi\left(x_{1}\right) \phi\left(x_{2}\right) \phi\left(x_{3}\right) \phi\left(x_{4}\right) e^{i \int d^{4} x \mathcal{L}_{*}(x)} \\
\quad \neq \int \prod_{x} \mathcal{D}(\phi(x)) \Lambda \triangleright\left(\phi\left(x_{1}\right) \phi\left(x_{2}\right) \phi\left(x_{3}\right) \phi\left(x_{4}\right)\right) e^{i \int d^{4} x \mathcal{L}_{*}(x)}
\end{aligned}
$$

showing that the measure is not twist-Poincaré invariant.

\section{$7 \quad$ Locality}

i) $\theta^{\mu \nu} \neq 0$, Untwisted Statistics:

The conventional quantization a scalar field on the noncommutative plane leads to nonlocal physics. However this non-locality is due to nonlocal interaction terms and does not show up in the free theory. As remarked earlier the free theory is identical to the scalar field theory for $\theta^{\mu \nu}=0$.

ii) $\theta^{\mu \nu} \neq 0$, Twisted Statistics:

The situation is quite different when one quantizes using twisted statistics. In this case, even the free theory is non-local. We have

$$
\begin{aligned}
& {[\Phi(x), \Phi(y)]=} \\
& \quad \int \frac{d^{3} p d^{3} k}{(2 \pi)^{3}\left(2 p_{0}\right)\left(2 k_{0}\right)}\left[e^{-i(p \cdot x+k \cdot y)}\left(1-e^{-i \theta^{\mu \nu} p_{\mu} k_{\nu}}\right) a_{p} a_{k}+e^{i(p \cdot x+k \cdot y)}\left(1-e^{-i \theta^{\mu \nu} p_{\mu} k_{\nu}}\right) a_{p}^{\dagger} a_{k}^{\dagger}\right. \\
& \quad+e^{-i(p \cdot x-k \cdot y)}\left\{\left(1-e^{i \theta^{\mu \nu} p_{\mu} k_{\nu}}\right) a_{p} a_{k}^{\dagger}-\left(2 p_{0}\right) \delta^{3}(p-k)\right\} \\
& \left.\quad+e^{i(p \cdot x-k \cdot y)}\left\{\left(1-e^{i \theta^{\mu \nu} p_{\mu} k_{\nu}}\right) a_{p}^{\dagger} a_{k}+\left(2 p_{0}\right) \delta^{3}(p-k)\right\}\right]
\end{aligned}
$$

This operator is not zero when $x$ and $y$ are space-like separated. For example, we can calculate it between two single-particle momentum eigenstates $|q\rangle$ and $|r\rangle$. We have

$$
\begin{aligned}
\langle q|[\Phi(x), \Phi(y)]| r\rangle & =\left(e^{i \theta^{\mu \nu} q_{\mu} r_{\nu}}-1\right)\left(e^{-i r \cdot x+i q \cdot y}-e^{i q \cdot x-i r \cdot y}\right) \\
& +\left(2 q_{0}\right) \delta^{3}(q-r)[D(x-y)-D(y-x)]
\end{aligned}
$$

where $D(x-y)$ was defined in (5.15). The last two terms together vanish for space-like separations, but the first term is in general nonzero for $q \neq r$. 
Although the free theory is (twisted) Poincaré invariant, it is non-local. Hence the spin-statistics theorem does not apply to it and there is no internal inconsistency coming from this theorem.

\section{On Twisted Tensor Product}

In [9], it has been suggested that the *-product and the twist of statistics are one and the same, and that considering both separately is what led to the result about the absence of UV-IR mixing.

We feel that this remark is incorrect. It is well-known in Hopf algebra theory [6] that the coproduct on a (quasi-triangular) Hopf algebra is associated with an " $R$-matrix" and that the latter fixes statistics. In our case, $R=\mathcal{F}^{-2}$ and that gives the representation of the permutation group via (3.13).

Incidentally, a "twisted" tensor product has been used in [9] in connection with the Drinfel'd twist. Its connection to the $*$-product is vague at best. It leads to twisted statistics, but not the correct one. We can see this as follows.

The "twisted" tensor product considered is

$$
\Phi_{0}^{(+)} \otimes_{T} \Phi_{0}^{(+)} \equiv e^{\frac{i}{2} \partial_{\mu} \otimes \theta^{\mu \nu} \partial_{\nu}} \Phi_{0}^{(+)} \otimes \Phi_{0}^{(+)}
$$

where the field $\Phi_{0}^{(+)}$is the creation part (say) of a free field constructed from the standard creation and annihilation operators in the usual manner. We have,

$$
\Phi_{0}^{(+)}(x) \Phi_{0}^{(+)}(y)=\Phi_{0}^{(+)}(y) \Phi_{0}^{(+)}(x)
$$

so that

$$
\begin{aligned}
\left(\Phi_{0}^{(+)} \otimes_{T} \Phi_{0}^{(+)}\right)(x, y) & =\left(e^{\frac{i}{2} \partial_{\mu} \otimes \theta^{\mu \nu} \partial_{\nu}}\right)\left(\Phi_{0}^{(+)} \otimes \Phi_{0}^{(+)}\right)(x, y) \\
& =\exp \left(\frac{i}{2} \frac{\partial}{\partial x^{\mu}} \theta^{\mu \nu} \frac{\partial}{\partial y^{\nu}}\right) \Phi_{0}^{(+)}(x) \Phi_{0}^{(+)}(y) \\
& =\exp \left(-\frac{i}{2} \frac{\partial}{\partial y^{\mu}} \theta^{\mu \nu} \frac{\partial}{\partial x^{\nu}}\right) \Phi_{0}^{(+)}(y) \Phi_{0}^{(+)}(x) \\
& =e^{-i \partial_{\mu} \otimes \theta^{\mu \nu} \partial_{\nu}}\left(\Phi_{0}^{(+)} \otimes_{T} \Phi_{0}^{(+)}\right)(y, x)
\end{aligned}
$$

This does not agree with (3.23).

Acknowledgments: The work of APB, BQ and AP is supported in part by DOE under grant number DE-FG02-85ER40231.

\section{References}

[1] M. Chaichian, P. P. Kulish, K. Nishijima and A. Tureanu, Phys. Lett. B 604, 98 (2004) arXiv:hep-th/0408069. 
[2] P. Aschieri, C. Blohmann, M. Dimitrijevic, F. Meyer, P. Schupp and J. Wess, Class. Quant. Grav. 22, 3511 (2005) arXiv:hep-th/0504183.

[3] R. Oeckl, Nucl. Phys. B 581, 559 (2000); arXiv:hep-th/0003018.

[4] A. P. Balachandran, G. Mangano, A. Pinzul and S. Vaidya, Int. J. Mod. Phys. A 21, 3111 (2006) arXiv:hep-th/0508002.

[5] A. P. Balachandran, A. Pinzul and B. A. Qureshi, Phys. Lett. B 634, 434 (2006) arXiv:hep-th/0508151.

[6] S. Majid, Foundations of Quantum Group Theory, Cambridge University Press, 1995.

[7] J. Zahn, Phys. Rev. D 73, 105005 (2006) arXiv:hep-th/0603231.

[8] A. P. Balachandran, A. M. Marques, A. R. Queiroz and P. Teotonio-Sobrinho, arXiv:hep-th/0608081.

[9] A. Tureanu, Phys. Lett. B 638, 296 (2006) arXiv:hep-th/0603219.

[10] R. Oeckl, Commun. Math. Phys. 217, 451 (2001); arXiv:hep-th/9906225. 